\title{
EXTRACTING AXIAL DEPTH AND TRAJECTORY TREND USING ASTIGMATISM, GAUSSIAN FITTING, AND CNNS FOR PROTEIN TRACKING
}

\author{
Kristofer delas Peñas ${ }^{1,3}$, Mariia Dmitrieva ${ }^{1}$, Joël Lefebvre ${ }^{1}$, Helen Zenner ${ }^{2}$, Edward Allgeyer ${ }^{2}$ \\ Martin Booth ${ }^{1}$, Daniel St Johnston ${ }^{2}$, Jens Rittscher ${ }^{1}$ \\ ${ }^{1}$ Department of Engineering Science, University of Oxford, Oxford, UK \\ ${ }^{2}$ Gurdon Institute, University of Cambridge, Cambridge, UK \\ ${ }^{3}$ Department of Computer Science, University of the Philippines, Quezon City, Philippines
}

\begin{abstract}
Accurate analysis of vesicle trafficking in live cells is challenging for a number of reasons: varying appearance, complex protein movement patterns, and imaging conditions. To allow fast image acquisition, we study how employing an astigmatism can be utilized for obtaining additional information that could make tracking more robust. We present two approaches for measuring the $z$ position of individual vesicles. Firstly, Gaussian curve fitting with CNN-based denoising is applied to infer the absolute depth around the focal plane of each localized protein. We demonstrate that adding denoising yields more accurate estimation of depth while preserving the overall structure of the localized proteins. Secondly, we investigate if we can predict using a custom CNN architecture the axial trajectory trend. We demonstrate that this method performs well on calibration beads data without the need for denoising. By incorporating the obtained depth information into a trajectory analysis, we demonstrate the potential of improving vesicle tracking.
\end{abstract}

Index Terms - tracking, biomedical imaging, gaussian fitting, denoising, convolutional neural networks, confocal microscopy

\section{INTRODUCTION}

The quantitative analysis of the vesicle movement is important for biological studies in cells and movement along $z$-axis can be essential for understanding biological processes. Several different approaches to achieve three-dimensional (3D) localization had been proposed in recent years $[1,2]$. These techniques mainly involve changing the shape of the point spread function (PSF) used in the imaging setup. Such techniques include astigmatism, double helix, and biplane [3].

MD, JL, HZ, EA, MB, and JR were funded by a Wellcome Collaborative award (203285) and D St J by a Wellcome Principal Research Fellowship (207496). JL was funded by a FRQNT postdoctoral fellowship (257844). JR was supported by EPSRC Seebibyte (EP/M013774/1). KdP was supported by EPSRC and MRC (EP/L016052/1), the University of the Philippines, and the Philippine Department of Science and Technology (ERDT).
Through PSF shape modification, the localization of single fluorescent molecules can be achieved not only in the lateral plane but also axially. Astigmatic localization microscopy is a popular single-molecule localization method, with several software variations and heavy usage in 3D localization competitions. The defining characteristic in images with astigmatism is the elongation of spots in one of the lateral axes as you move from the focal plane towards the objective, and elongation in the other lateral axis as a particle moves away from the focal plane and the objective. The usual way to extract depth with this kind of data is to fit an elliptical Gaussian curve to the localized spots [3]. This Gaussian fitting process is favored because of its simplicity and the speed by which it can be computed. However, due to non-ideal imaging optics and background noise, Gaussian fitting may sometimes fail to obtain good depth estimates.

Localization and tracking algorithms were demonstrated to work really well in several settings. However, these often require the data to be collected over a long period of time to produce images that have high signal-to-noise ratio (SNR). In the case of live imaging and capturing the movement of molecules like proteins, we would often trade off SNR with increased temporal resolution. In such cases, the noise in the collected data poses a challenge in processing and analysis.

In this paper, we utilize two approaches for astigmatic localization: standard Gaussian curve fitting and a CNN-based model to classify the axial trajectory trend of the vesicles. The first approach provides quantitative results in $z$-localization. It uses the standard Gaussian fitting method. We improve on this by applying a denoising step to get good depth estimates even on noisy images. We also present a second approach that doesn't require denoising and is based on the temporal changes in the vesicle appearance. We modelled and tested these approaches using astigmatic spinning disk confocal microscopy images of calibration beads. The contributions of the paper are the application of a CNN-based self-supervised denoising step to address the low SNR in confocal imaging to achieve better depth estimations through Gaussian fitting and the development of a lightweight $\mathrm{CNN}$-based approach 
to classify the axial trajectory trend. Lastly, we demonstrate how the depth information extracted from the two approaches can be used to improve the association of localized molecules for protein tracking on images of living epithelial cells of Drosophila egg chamber as described in [4].

\section{METHODOLOGY}

\subsection{Asymmetric Gaussian fitting}

The estimation of the point spread function using Gaussian fitting on astigmatic conditions is powerful enough to estimate depth with $50 \mathrm{~nm}$ precision $[1,5]$. In general, the standard deviations of a Gaussian curve along the two lateral axes are modelled with the following equation:

$$
f(x, y)=A e^{-\left(\frac{\left(x-x_{0}\right)^{2}}{2 \sigma_{x}^{2}}+\frac{\left(y-y_{0}\right)^{2}}{2 \sigma_{y}^{2}}\right)}+B,
$$

where $A$ is the intensity or photon count of the Gaussian peak, $x_{0}$ and $y_{0}$ are the spatial coordinates of the peak, $\sigma_{x}$ and $\sigma_{y}$ are the standard deviations along the $x$ and $y$ axes, and $B$ is an offset term for the background fluorescence.

In our experiments, we imaged living epithelial cells of Drosophila egg chambers using a spinning disk confocal microscope with 0.97 radians peak-to-peak of astigmatism. We adapted the Gaussian fitting method to use the $x$ - $y$ localization obtained from the approach described in [4]. For each $x-y$ localization, we extracted a $16 \times 16$ region of interest, with the localized spot in the center, and individually fitted Gaussian curves to obtain the values for $\sigma_{x}$ and $\sigma_{y}$.

Next, using the calibration beads data and through nonlinear least square optimization using the Levenberg-Marquardt algorithm as provided in scipy [6] optimization library, we model a defocus curve described in [5] as follows:

$$
\frac{\sigma_{x}}{\sigma_{y}}=\frac{\sqrt{1+\left(\frac{z-c_{x}}{d}\right)^{2}+A_{x}\left(\frac{z-c_{x}}{d}\right)^{3}+B_{x}\left(\frac{z-c_{x}}{d}\right)^{4}}}{\sqrt{1+\left(\frac{z-c_{y}}{d}\right)^{2}+A_{y}\left(\frac{z-c_{y}}{d}\right)^{3}+B_{y}\left(\frac{z-c_{y}}{d}\right)^{4}}}
$$

where $d$ is the depth of focus, $c_{x}$ and $c_{y}$ are the lateral offsets, and $A_{x}, A_{y}, B_{x}, B_{y}$ are coefficients of higher order terms to correct for non-ideal optics.

One challenge in this approach, however, is the amount of noise in regions of interest. Fitting a 2D Gaussian on these noisy images yields inaccurate values of $\sigma_{x}$ and $\sigma_{y}$ and therefore produces poor estimates of the axial coordinates. To resolve this issue, we employed a deep learning algorithm to denoise the noisy confocal images. Noise2self [7] is a selfsupervised CNN-based algorithm that has been demonstrated to successfully denoise natural and microscopy images. In the absence of ground truth reference data for the confocal images, a self-supervised technique like Noise2self is a suitable method for denoising. This algorithm assumes the statistical independence of the noise across pixels to calibrate the
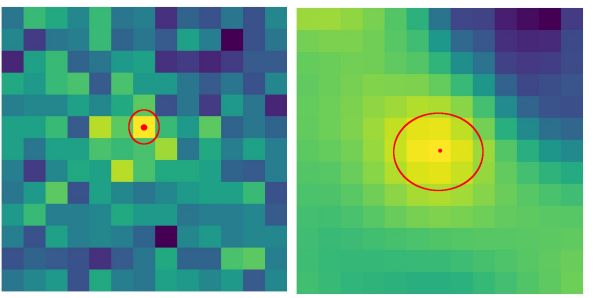

Fig. 1: Using Noise2self. (a) Protein localized with the bright spot at the center. (b) Noise2Self denoising result of the same protein.

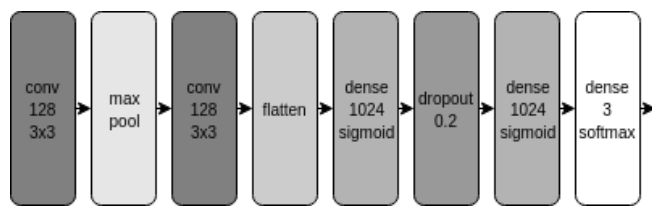

Fig. 2: Custom CNN architecture used for the classification of axial trajectory trend

hyperparameters of a median filter or CNNs for denoising. A model consisting of the original implementation of Noise 2self and a Densely Connected Convolutional Network (DCN) [8] as backend was used to suppress noise from the confocal microscopy data prior to Gaussian fitting. This additional step resulted in a more accurate localization of the spots and a better estimation of the Gaussian curves. Figure 1 illustrates an example of a localized protein before and after denoising.

\subsection{Classifying protein axial movement trend using CNN}

In contrast to the Gaussian fitting based approach where the depth information is extracted from a single frame, this CNNbased approach exploits temporal information to extract the trend in the axial trajectory (upward, downward, constant). This approach does not require any kind of denoising and the trend information can be sufficient for some applications. With this, we formulate the protein axial tracking as a threeclass classification problem. Using the $x-y$ localization from [4], we obtain $16 \times 16$ regions of interest, centered on the localized spots. For each localized protein, we stack the ROIs from three successive frames to form a three-channel image that serves as input to the CNN classifier trained for this task.

For the CNN classifier, we utilized a lightweight custom architecture, shown in Figure 2. We trained this network implemented in Keras using the following parameters: Adam optimizer with learning rate (10e-5), batch size of 32, 1000 epochs, and categorical cross entropy as loss function.

The training data is obtained from calibration beads videos acquired with the same spinning disk confocal microscope setup as the vesicle tracking movies. We simulated the upward, downward, and constant movement trend by getting sets of three frames, each from a different depth in the 
$z$-stack, and stacked the frames to construct three-channel input. Overall, we have constructed 64,092 three-channel input data and used a 50-50 train-test split. To make the model more robust, we performed data augmentation through addition of Gaussian noise with $\mu$ of 5.0 and $\sigma$ of 10.0.

\section{RESULTS AND DISCUSSION}

In this section, we evaluated the performance of the two approaches. We used the original calibration beads data imaged with a spinning disk confocal microscope with astigmatism. $Z$-stacks of the calibration beads were taken with 31 steps and $50 \mathrm{~nm}$ step size. Two $z$-stacks were obtained, one imaged with $1 / 1000$ th exposure time relative to the other, yielding one with low SNR and one with high SNR.

\subsection{Asymmetric Gaussian fitting}

To validate the results for the Gaussian fitting with denoising approach and to ensure that no artifacts are introduced in denoising and the measurements obtained are correct, we tested the same denoising model on the low SNR $z$-scan of calibration beads with known axial coordinates. The calibration beads were imaged with the same conditions as the protein data, with the same camera frame rate to obtain similar level of noise. We observed that the denoising approach preserves the overall structure of the spots. Figure 3 illustrates the advantage of the denoising for the Gaussian fitting. Denoising before Gaussian fitting yields better estimated ratio of $\sigma_{x} / \sigma_{y}$ when compared with noisy data. Without denoising, the $x-y$ standard deviation ratios we obtain from fitting are almost constant, regardless of depth. In other cases, the computed values are way off from the true values as shown in Figure 4. This indicates that we cannot obtain useful depth information as the noise obscures the fluorescent signal, but with the denoising step, we can decrease the amount of noise that will result to a better fit. Quantitatively, adding the denoising step translated to a ten-fold decrease (from 0.0110 to 0.0013 ) in the mean squared error (MSE) of the fit with respect to the high SNR data. However, the problem still remains towards the ends of the $z$ range $( \pm 500 \mathrm{~nm}$ and further), far away from the focal plane, where we observed an MSE of 0.1361 , but this can be attributed to increased loss of signal in depths very far from focus.

\subsection{Classifying protein axial movement trend using $\mathrm{CNN}$}

Overall, the CNN-based approach to classify the axial trajectory trend provides very encouraging results. With an early stopping criterion set with minimum improvement delta of $10 \mathrm{e}-7$, training converged early at epoch 40 , with 0.0361 loss and $98.91 \%$ accuracy. We evaluated the model with the remaining $50 \%$ of the data, and found the model performing well at $98.64 \%$ test accuracy.

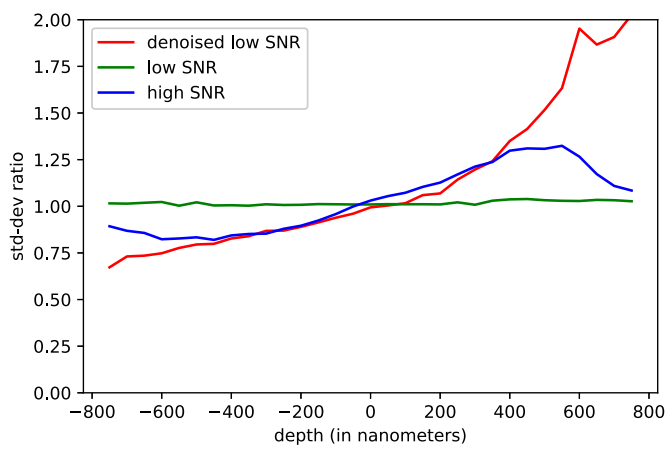

Fig. 3: Gaussian fitting on denoised low SNR data resulted in better depth approximations.

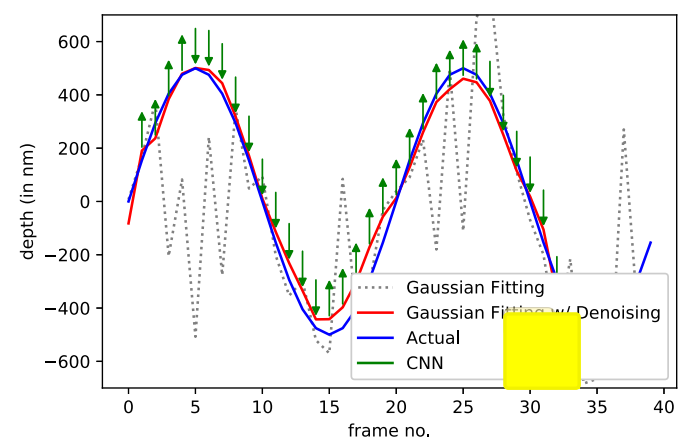

Fig. 4: Test results on calibration beads fixed on $x-y$ with the sample stage moved in a sinusoid manner in $z$.

To further evaluate this annroach, we captured additional calibration beads data by mc the sample stage in a sinusoid manner in the $z$ directic.., ..mulating the up and down motion of localized molecules. Both approaches were tested on the data and Figure 4 illustrates the results, providing the comparison of the proposed CNN-based approach and Gaussian fitting with denoising to the actual depths.

\subsection{Using extracted depth information in protein track- ing}

Lastly, we illustrate the advantage of the proposed depth estimators to improve tracking accuracy. Here, we utilize the approach presented by Dmitrieva and collaborators [4] which introduced a two-step linking process for data association. Firstly, a set of short tracks (tracklets) are formed based on the distance between the detections. Secondly, the tracklets are linked to form final tracks. The linking is based on the tracklets' parameters and defined by the connectivity score. The score represents a probability of the tracklet pair to be connected and is computed by inference over a Bayesian net- 

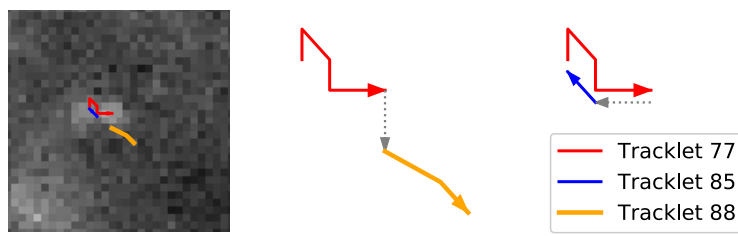

Fig. 5: Example of tracklets generated from [4]. Tracklets 85 and 88 are possible connections to track 77 . With the Bayesian Network in [4], tracklet 85 is more likely to be connected.

\begin{tabular}{l|l|l|l|l}
\hline & \multicolumn{2}{|c|}{ TRACKLET 88 } & \multicolumn{2}{c}{ TRACKLET 85 } \\
\hline & w/o & w/ & w/o & w/ \\
& depth & depth & depth & depth \\
TRACKLET 77 & 0.8536 & 0.8536 & 0.9325 & $\mathbf{0 . 9 6 2 5}$ \\
\hline
\end{tabular}

Table 1: Connectivity matrix for tracklet 77 with and without depth depth estimation for tracklets 88 and 85 .

work (BN).

We applied the tracking approach on the protein data with astigmatism and compared the connectivity scores for tracklets with and without the extracted depth information using the two proposed approaches. Intuitively, we expect an increase in the likelihood of connectivity for tracklets whose ends are more or less at the same depth or following the same trajectory trend. Figure 5 illustrates an example of the tracklinking task with three tracklets to be considered. Using the originally proposed tracking solution without depth information, tracklet 77 is linked with tracklet 85 (and not 88) with score of 0.9325 . Using the approaches presented in this paper, we estimated tracklet 77 to be moving downwards $(647 \mathrm{~nm}$ depth) and connecting tracklet 85 continues this trend (679 $\mathrm{nm}$ depth) but not tracklet 88 (-274 nm depth). As presented in Table 1, if we include this depth information and modify the BN topology in [4] to incorporate extracted depth information, we still see similar patterns in the connectivity matrix for tracklet 77 but with an increase in the magnitude on the tracklet to be connected. Adding depth information from the proposed approaches strengthens this track linking process by increasing the confidence in connectivity. For cases with close connectivity scores, the depth information may be crucial in correctly linking tracks.

\section{CONCLUSION}

Two approaches for extracting depth from confocal microscopy data with astigmatism are presented. The first approach uses the standard and widely accepted Gaussian fitting method. We added a denoising preprocessing step and demonstrated that this additional step resulted in better depth estimates. The CNN-based approach focuses on temporal changes in the particle appearance and provides axial trajectory trend. We have shown that even with a lightweight architecture, this information can be obtained without the need for denoising.

The CNN-based approach could be extended to finergrained classes. For example, quantitative evaluation of the movement in $z$-axis, as an extension of the axial trajectory trend. This would give insight on the speed by which the molecules move in the $z$ direction. For both approaches, their integration with existing 2D localization and tracking algorithms like in [4] and further validation tests may provide better models to understand underlying subcellular mechanisms.

\section{REFERENCES}

[1] S. Holden, S. Uphoff, and A. Kapanidis, "Daostorm: An algorithm for high-density super-resolution microscopy," Nature methods, vol. 8, pp. 279-80, 042011.

[2] M. Ovesný, P. Křížek, J. Borkovec, Z. Švindrych, and G. M. Hagen, "ThunderSTORM: a comprehensive ImageJ plug-in for PALM and STORM data analysis and super-resolution imaging," Bioinformatics, vol. 30, pp. 2389-2390, 052014.

[3] D. Sage, T.-a. Pham, H. Babcock, T. Lukes, T. Pengo, J. Chao, R. Velmurugan, A. Herbert, A. Agrawal, S. Colabrese, A. Wheeler, A. Archetti, B. Rieger, R. Ober, G. Hagen, J.-B. Sibarita, J. Ries, R. Henriques, M. Unser, and S. Holden, "Super-resolution fight club: Assessment of $2 \mathrm{~d} 3 \mathrm{~d}$ single-molecule localization microscopy software," Nature Methods, vol. 16, 052019.

[4] M. Dmitrieva, H. L. Zenner, J. Richens, D. S. Johnston, and J. Rittscher, "Protein tracking by cnn-based candidate pruning and two-step linking with bayesian network," in IEEE International Workshop on Machine Learning for Signal Processing, 2019.

[5] B. Huang, W. Wang, M. Bates, and X. Zhuang, "Threedimensional super-resolution imaging by stochastic optical reconstruction microscopy," Science (New York, N.Y.), vol. 319, pp. 810-3, 032008.

[6] E. Jones, T. Oliphant, P. Peterson, et al., "SciPy: Open source scientific tools for Python," 2001-. [Online; accessed 7 October 2019].

[7] J. Batson and L. Royer, "Noise2self: Blind denoising by self-supervision," CoRR, vol. abs/1901.11365, 2019.

[8] G. Huang, Z. Liu, and K. Q. Weinberger, "Densely connected convolutional networks," CoRR, vol. abs/1608.06993, 2016. 
final:EXTRACTING AXIAL DEPTH AND TRAJECTORY

TREND USING ASTIGMATISM , GAUSSIAN FITTING , AND

CNNS FOR PROTEIN TRACKING Kristofer delas Pe Department of Engineering Science, University of

Oxford , Oxford , UK Gurdon Institute , University of

Cambridge ,

Dmitrieva, Mariia; Zenner, Helen; Allgeyer, Edward; Booth, Martin;

Johnston, Daniel St; Rittscher, Jens

01 Mariia Dmitrieva

Page 1

22/1/2020 11:28

we demonstrate potential improvement of the vesicle tracking

02 Mariia Dmitrieva

Page 3

22/1/2020 11:38

mention in the capture Gaussian fitting (GF) and you can reduce the legend to:

GF w/o denoising

GF $w$ denoising

03 Mariia Dmitrieva

Page 3

22/1/2020 11:39

these approaches

04 Mariia Dmitrieva

Page 3

22/1/2020 11:45

and Gaussian fitting with and without denoising step to the actual depth. It can be seen that both approaches provide realiable results and can be used for the depth evaluation with the noisy data. 